\title{
Erratum to: Genetic mapping and QTL analysis of Botrytis resistance in Gerbera hybrida
}

\author{
Yiqian Fu • Alex van Silfhout • Arwa Shahin • Ronny Egberts • Martin Beers • \\ Ans van der Velde • Adrie van Houten • Jaap M. van Tuyl • Richard G. F. Visser • \\ Paul Arens
}

Published online: 17 March 2017

C) Springer Science+Business Media Dordrecht 2017

Erratum to: Mol Breeding (2017) 37: 13

DOI 10.1007/s11032-016-0617-1

Unfortunately, in the original version of this article part of Fig. 1 was deleted in the final proofing step.

The missing part of Fig. 1 with linkage groups 18-24.

The online version of the original article can be found at http://dx. doi.org/10.1007/s11032-016-0617-1

Y. Fu • A. van Silfhout - A. Shahin · J. M. van Tuyl •

R. G. F. Visser · P. Arens $(\square)$

Plant Breeding, Wageningen University \& Research, PO Box 386, 6700AJ Wageningen, The Netherlands

e-mail: paul.arens@wur.nl

R. Egberts · A. van Houten

Schreurs Holland B.V., Hoofdweg 81, 1424PD De Kwakel,

The Netherlands

M. Beers · A. van der Velde

Florist Holland B.V., Dwarsweg 15, 1424PL De Kwakel,

The Netherlands 
Fig. 1 Consensus linkage map of gerbera, LG18-LG24

\section{LG18}

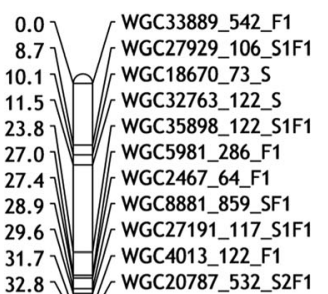

32.8 WGC20787_532_S2F1

35.4 - WGC4372_144_S1F

36.7 WGC59_150_S1F2

38.7 WGC2644_234_S1

39.0 - WGC7433_150_S2F1a

40.1

42.9 UWGC8743_118_S1F1b

42.9 WGC27592_275_S2F2

44.7 - WGC8743_118_S1F1

45.2 - - WGC18800_256_S1F1

47.3 | - WGC19040_355_F2b WGC19040_355_F2b

$\left.\begin{array}{l}47.4 \\ 47.9\end{array}\right]|-|\left[\begin{array}{l}\text { WGC8743_118_S1F1a } \\ \text { WGC19782_600_SF2 }\end{array}\right.$

$\left.\begin{array}{l}47.9 \\ 56.8\end{array}\right] \mid-\left[\begin{array}{l}\text { WGC19782_600_SF2 } \\ \text { WGC6074_441_S2F }\end{array}\right.$

$\begin{aligned} & 60.5 \\ & 60.7\end{aligned} \mid \|\left[\begin{array}{l}\text { WGC6074_441_S2F } \\ \text { WGC35264_283_S2F1 }\end{array}\right.$

60.7 WGC8954_249_S2F

64.8 || WGC34991_187_S2F1

71.0 $]$ WGC5549_561 S2

75.6 ] WGC17619_188_S2

80.2 ] WGC7433_150_S2F1

81.3 WGC9144_292_S2

89.7 [WGC12786_448_S

\section{LG21}

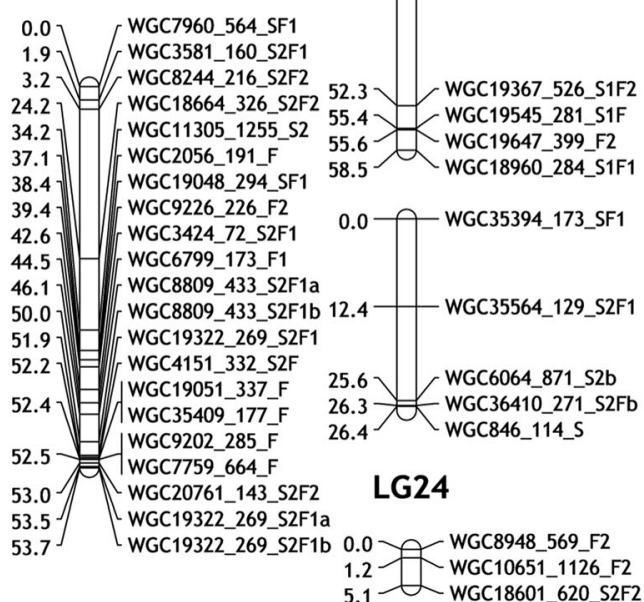

LG19

12.8 $\int \begin{aligned} & \text { WGC17038_450_F } \\ & \text { WGC2951_238_S2 }\end{aligned}$

15.7 WGC25353_140_S

21.7 WGC34570_147_S2F

26.0] WGC34116_168_F

26.2 _WGC17332_1876_S1F

32.2 WGC19112_269_SFa

33.3 WGC4251_260_Sa

34.3 $=$ WGC19112_269_SFb

34.8 WGC4251_260_Sb

45.0 \WGC24446_221_S1F2

46.4 WGC4399_409_S

46.6 WGC33922_118_SF2

48.5 -WGC34858_1542_SF1

49.7 -WGC7762_411_F

$64.5-W G C 4953$-374_S1

\section{LG22}

0.0 WGC35300 107 Fb 0.6 WGC9248_106_S1F1 3.3 WGC35300_107_Fa

4.4 WGC36410_271_S2F

9.2 WGC7516_117_F

13.3 WGC35564_129_S2F1b

15.3 WGC35564_129_S2F1a

19.0 WGC35185_192_F1

24.9 WGC35394_173_SF1a

25.7 WGC35394_173_SF1b

WGC18601 620 S2F2

\section{LG20}

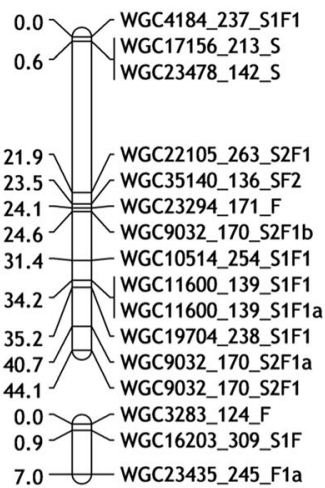

\section{LG23}

0.0 ] WGC22447_285_Fb

4.8 ] WGC7127_127_S1F2

$5.3]$ WGC1067_494_S1

5.9 WGC15405_1293_S1F

7.8 $=$ WGC22447 285 F

7.9 WGC1084_721_F

18.8 - WGC27629_93_S

27.4 WGC1824_721_S1F1

27.6 WGC407_4995_S1F1

29.6 WGC16170_118_F1

29.8 WGC19020 660 S1

30.1 - WGC3454 459 F1

30.2 - WGC3745_120_S1

31.1 U WGC21545_548_S1F1

32.0 [WGC3454 459 F1a

60.1 -WGC22355_237_S1F1

61.3 WGC34390_211

61.5 WGC8574_435_S1F

61.7 WGC7513_582_S1F

62.4 \WGC8047_200_F1

63.0 [WGC14274 384 F1

(

90.0-WGC27795_176_F

0.0 WGC35400_175_F1

0.6 WGC3581_160_S2F1

F WGC18015_419_F1

0.8 WGC18469_69_F1

WGC14461_576_S2F1 